\title{
Severe Hypoglycemic Coma Event on MRI: Specific Brain Necrosis
}

\author{
Tong $\mathrm{DM}^{1,2^{*}}$ and Zhou $\mathrm{YT}^{3}$ \\ ${ }^{1}$ Department of Neurology, Affiliated Shuyang People' Hospital, XuZhou Medical College, Jiangsu, China \\ ${ }^{2}$ Departments of Neurology, the Affiliated Pingxiang Hospital, Southern Medical University, China \\ ${ }^{3}$ Affiliated Shuyang People' Hospital, XuZhou Medical College, Jiangsu, China
}

*Corresponding author: Tong DM, Department of Neurology, Affiliated Shuyang People' Hospital, XuZhou Medical College, Yingbin Road, Shu town,Jiangsu, China, E-mail: tongdaoming@163.com

Citation: Tong DM, Zhou YT (2014) Severe hypoglycemic coma event on MRI: specific brain necrosis. SAJ Case Rep 1: 104. doi: 10.18875/2375-7043.1.104

Article history: Received: 02 August 2014, Accepted: 13 August 2014, Published: 14 August 2014

\begin{abstract}
With the widespread of sale and use of oral hypoglycemic drugs or insulin, hypoglycemic coma event have become a common medical emergency. Some patients with severe hypoglycemic coma led to prolonged hypoglycemia with fatal coma due to delay in the treatment time [1], or may led to coma, reasons are difficult to assess as the blood sugar have returned to normal levels. Here, we report a case of severe hypoglycemic coma events, blood sugar level has returned to normal and its brain MRI changes.
\end{abstract}

Keywords: Hypoglycemic; Brain Necrosis; MRI

\section{Case report}

A 76-year-old woman with hypertension was in coma for $48 \mathrm{hrs,} \mathrm{and} \mathrm{its} \mathrm{bed} \mathrm{is} \mathrm{exposed} \mathrm{to} \mathrm{anti-diabetic} \mathrm{drugs,} \mathrm{i.e.,} \mathrm{glibenclamide}$ 100 tables/bottle leaving only 90 tables $(2.5 \mathrm{mg} /$ piece). The patient had no exposure to carbon monoxide, and no history of diabetes mellitus and cardiac arrest. She used to live alone, the patient was unconscious for two days, only then discovered she fell ill, and then she was given $50 \mathrm{ml}$ of sugar water oral. Unfortunately, the patient remained in a coma. Brain CT scans were normal. Blood pressure was 153/92 $\mathrm{mmHg}$. The respiratory rate and SpO2 were normal. She had a Glasgow coma score (GCS) of E1M4V1 with symmetry pupils (diameter $1.5 \mathrm{~mm}$ ), no light reflex, corneal reflex, and extraocular movements. On admission, laboratory blood glucose was $8.6 \mathrm{mmol} / \mathrm{l}$. Electrolytes, liver and renal function was normal. ECG revealed normal. On the second day of hospital admission, brain DWI revealed diffuse high signals on the cerebral cortex bilaterally, hippocampus, and basal ganglia (Figure 1 A and B). After four days of admission, the patient was still in deep coma and phlegm, so the tracheotomy breathing was performed. After five days, the patient was into a vegetative state, and she was discharged after 21 days. On follow-up 2 months later, the patient was a persistent vegetative state.
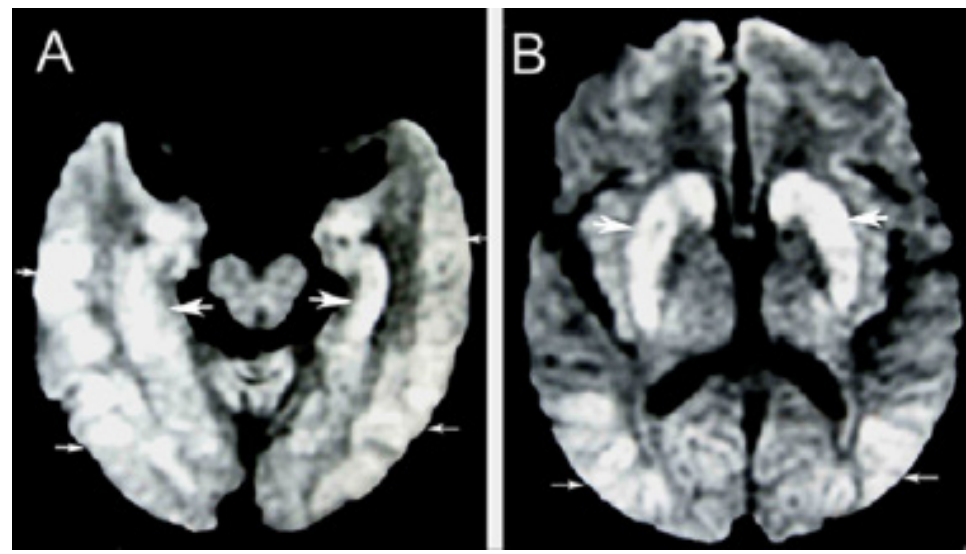

Figure 1: Brain DWI in patient with hypoglycemic coma due to the mistake of overdose glibenclamide. On the third'day of coma, brain DWI revealed diffuse high signals in the bilateral hippocampus (A. thick arrows), temporal and occipital cortex (A. tiny arrows); simultaneously in bilateral basal ganglia (B. thick arrows), and occipital cortex gray matter (B. tiny arrows). 


\section{Conclusion}

Hypoglycemic coma has become a common medical emergency, it is not only a common complication of diabetes due to widely available for use of oral hypoglycemic agents or insulin, but also can be a cause of mistake or even suicide. Some serious hypoglycemic patients often delay in treatment time led to irreversible coma [1] or because the half-life of hypoglycemic agents is past, and the blood sugar has been returned to normal levels, leading to coma reasons difficult to assess or misdiagnosed.

The patient shows neither signs of cardiac arrest, nor a history of exposure to carbon monoxide, so the whole brain anoxic and hypoxic-ischemic encephalopathy can be excluded. In general, if the cerebral circulation is stopped (more than 5 minutes), ischemia and hypoxia, can produce localized neuronal apoptosis and necrosis. It is difficult to fully recover in $6 \mathrm{~h}$. Cerebral ischemia may be focal or global. If patient was in cerebral ischemia, MR imaging showed that the global ischemia is usually in the gray matter junctions [2] whereas focal infarctions is in regions between major arterial territories [3]. Moreover, infarction is almost multiple asymmetric (different sizes), and also with the characteristics of gray matter involvement. Although infarctions in the bilateral basal ganglia is not uncommon, but it is asymmetry, and also in other vascular territory, including the brain's white matter areas, often with ischemic lesions, which may be termed "satellite lesions". Thus, the current MR image change is not used a global ischemic or focal infarction to explain.

Predominantly gray matter damage is termed "selective neuronal necrosis" [4]. Our patient's DWI showed only a symmetrical gray matter injury, including bilateral temporal occipital cortex, basal ganglia, and hippocampus; whereas white matter not be affected. This selective neuronal necrosis in the gray of brain on DWI is a specific feature hypoglycemic brain injury [5,6]. However, glucose deprivation is different than the cerebral circulation stops. Coma can occur at glucose levels in the range of 2.3-2.7 mmol/1 [7]. Experiments confirmed that some of the neuronal apoptosis can occur after hypoglycemic coma lasting 30 minutes [8], whereas glucose levels in the range of 1.7-1.9 mmol/l can lead to a prolonged coma due to irreversible neuronal death. In studies of insulininduced hypoglycemia in monkeys, 5-6 hours of blood glucose concentrations of less than $1.1 \mathrm{mmol} / \mathrm{L}$ were required for the regular production of neurological damage [9]. Profound, prolonged hypoglycemia can cause brain death [8]. It can be seen, the irreversible of brain injury is associated with the severity and duration of hypoglycemia coma [10]. Thus, delayed correction of plasma glucose concentration does not interrupt this cell death process. Therefore, the monitoring of the brain MR image is useful for the diagnosis of coma $[9,11]$. Our patient was an old woman lived alone and with a history of exposure to glibenclamide (half-life of 12 hours). She was conscious for two days when discovered she fell ill, and she was given oral $50 \mathrm{ml}$ of glucose water before admission. On admission, although her blood glucose was $8.6 \mathrm{mmol} / \mathrm{l}$, her prolonged coma was a performance of severe hypoglycemic brain lesion. Moreover, this specific high signals in the bilateral cerebral cortex and hippocampus, and especially in bilateral basal ganglia due to severe hypoglycemic event has been confirmed by our MR-DWI.

On other hand, the analysis of the brain MR image' changes after a hypoglycemia coma can be useful to predict the prognosis. For instance, some prolonged hypoglycemia coma associated with lesions of the internal capsule, corona radiata and cortex have been reversed by raising glucose [12-14]. Lesions of the bilateral basal ganglia may survive in a persistent vegetative state, [5,6] even with a poor outcome and death $[13,15]$.

According to the diagnostic criteria of persistent vegetative state published by the American Academy of Neurology in 1995, three months later, the patient with a persistent vegetative state has been diagnosed. This suggests that these high signals on DWI from severe hypoglycemia due to the mistake of overdose hypoglycemic drugs lead to irreversible neuronal necrosis or death. Patient could be misdiagnosed if blood glucose was returned to a normal level due to the half-life of hypoglycemic drugs absented. Therefore, we recommend that comatose patient who showed the specific high signals in the gray of brain on DWI, should be the evidence of diagnosed severe hypoglycemic coma.

\section{References}

1. Daggett P, Nabarro J (1984) Neurological aspects of insulinomas. Postgrad Med J 60: 577-81.

2. Arbelaez A, Castillo M, Mukherji SK (1999) Diffusion-weighted MR imaging of lobal cerebral anoxia. AJNR Am J Neuroradiol 20 : $999-1007$.

3. Castillo M (1998) Imaging of cerebral infarction. Curr Probl Diagn Radiol 27: 105-32.

4. Rothman SM, Olney JW (1986) Glutamate and the pathophysiology of hypoxic/ ischemic brain damage. Ann Neurol 19: 105-11.

5. Fujioka M, Okuchi K, Hiramatsu KI, Sakaki T, Sakaguchi S, et al. (1997) Specific changes in human brain after hypoglycemic injury. Stroke 28 : 584-7.

6. Lim CC, Gan R, Chan CL, Tan AW, Khoo JJ, et al (2009) Severe hypoglycemia associated with an illegal sexual enhancement product adulterated with glibenclamide: MR imaging findings. Radiology 250: 193-201.

7. Ben-Ami H, Nagachandran P, Mendelson A, Edoute Y (1999) Drug-induced hypoglycemic coma in 102 diabetic patients. Arch Intern Med 159: 281-4.

8. Ouyang YB1, He QP, Li PA, Janelidze S, Wang GX, et al. (2000) Is neuronal injury caused by hypoglycemic coma of the necrotic or apoptotic type? Neurochem Res 25: 661-7.

9. Tkacs NC, Pan Y, Raghupathi R., Dunn-Meynell AA, Levin BE (2005) Cortical Fluoro-Jade staining and blunted adrenomedullary response to hypoglycemia after noncoma hypoglycemia in rats. J Cereb Blood Flow Metab 25: 1645-55.

10. Philip EC (2007) Hypoglycemia, functional brain failure, and brain death. J Clin Invest 117: 868-70.

11. Kahn KJ, Myers RE (1971) Insulin-induced hypoglycaemia in the non-human primate. I. Clinical consequences. William Heinemann Medical Books Ltd. London, United Kingdom: 185-194. 
12. Jung SL, Kim BS, Lee KS, Yoon KH, Byun JY (2005) Magnetic resonance imaging and diffusion-weighted imaging changes after hypoglycemic coma. J Neuroimaging 15: 193-6.

13. Lo L, Tan AC, Umapathi T, Lim CC (2006) Diffusion-weighted MR imaing in early diagnosis and prognosis of hypoglycemia. AJNR 27: 1222-4.

14. Mori F, Nishie M, Houzen H, Yamaguchi J, Wakabayashi K (2006) Hypoglycemic encephalopathy with extensive lesions in the cerebral white matter. Neuropathology 26: 147-52.

15. Finelli PF (2001) Diffusion-weighted MR in hypoglycemic coma. Neurology 57: 933-5. 